IPNO-DR-05-06

\title{
Quark-antiquark bound state equation in the Wilson loop approach with minimal surfaces ${ }^{1}$
}

\author{
F. Jugeau ${ }^{a}$ and H. Sazdjian ${ }^{b}$ \\ ${ }^{a}$ Instituto de Fisica Corpuscular, IFIC, \\ Edificio Institutos de Investigacion, Apt. de Correus 22085, \\ E-46071 València, Spain \\ E-mail: frederic.jugeau@ific.uv.es \\ ${ }^{b}$ Institut de Physique Nucléaire ${ }^{2}$, Groupe de Physique Théorique, \\ Université Paris XI, F-91406 Orsay Cedex, France \\ E-mail: sazdjian@ipno.in2p3.fr
}

\begin{abstract}
The quark-antiquark gauge invariant Green function is studied through its dependence on Wilson loops. The latter are saturated, in the large- $N_{c}$ limit and for large contours, by minimal surfaces. A covariant bound state equation is derived which in the centerof-mass frame and at equal-times takes the form of a Breit-Salpeter type equation. The large-distance interaction potentials reduce in the static case to a confining linear vector potential. In general, the interaction potentials involve contributions having the structure of flux tube like terms.
\end{abstract}

PACS numbers: 03.65.Pm, 11.10.St, 12.38.Aw, 12.38.Lg, 12.39.Ki.

Keywords: QCD, Confinement, Wilson loop, Minimal surfaces, Bound states, Quarkonium.

\footnotetext{
${ }^{1}$ Talk given by H.S. at the Workshop QCD at Work 2005, Conversano, Italy, 16-20 June 2005.

${ }^{2}$ Unité Mixte de Recherche 8608.
} 
The Wilson loop [1] is defined as the trace in color space of the path-ordered phase factor of the gluon field on a closed contour $C$ :

$$
\Phi(C)=\frac{1}{N_{c}} \operatorname{tr}_{c} P e^{-i g \oint_{C} d x^{\mu} A_{\mu}(x)} .
$$

Its vacuum expectation value, denoted $W(C)$,

$$
W(C)=\langle\Phi(C)\rangle
$$

is a functional of the contour $C$. Loop equations were obtained and studied by Polyakov 2] and Makeenko and Migdal [3, 4, 5. The Wilson loop essentially satisfies two types of equation, which are equivalent to the QCD equations of motion: The Bianchi identity and the loop equations (or Makeenko-Migdal equations). A third property, factorization, is obtained in the large- $N_{c}$ limit [6] for two disjoint contours: $W\left(C_{1}, C_{2}\right)=W\left(C_{1}\right) W\left(C_{2}\right)$.

Further simplification is obtained in the large- $N_{c}$ limit of the theory. In that limit, for large contours, i.e., at large distances, nonperturbative asymptotic solutions to the Wilson loops are represented by the minimal surfaces having as supports the loop contours [3. 7. Therefore, if one is interested only in the large-distance properties of the theory, saturation of the Wilson loop averages by minimal surfaces provides a correct description of the theory in this regime. In that case, the Wilson loop average can be represented by the following functional of the contour $C$ :

$$
W(C)=e^{-i \sigma A(C)},
$$

where $\sigma$ is the string tension and $A(C)$ the minimal area with contour $C$.

Minimal surfaces also appear as natural solutions to the Wilson loop averages in twodimensional gauge theories [8].

To deal with the quarkonium bound state problem, one starts with the two-particle gauge invariant Green function for quarks $q_{1}$ and $q_{2}$ with different flavors and with masses $m_{1}$ and $m_{2}$ :

$$
G\left(x_{1}, x_{2} ; x_{1}^{\prime}, x_{2}^{\prime}\right) \equiv\left\langle\bar{\psi}_{2}\left(x_{2}\right) U\left(x_{2}, x_{1}\right) \psi_{1}\left(x_{1}\right) \bar{\psi}_{1}\left(x_{1}^{\prime}\right) U\left(x_{1}^{\prime}, x_{2}^{\prime}\right) \psi_{2}\left(x_{2}^{\prime}\right)\right\rangle_{A, q_{1}, q_{2}} .
$$

Here, $U\left(x_{2}, x_{1}\right)$ is the path-ordered phase factor,

$$
U\left(x_{2}, x_{1}\right)=P e^{-i g \int_{x_{1}}^{x_{2}} d z^{\mu} A_{\mu}(z)},
$$

taken along the straight-line $x_{1} x_{2}$ (and similarly for $U\left(x_{1}^{\prime}, x_{2}^{\prime}\right)$ ). Integrating in the large- $N_{c}$ limit with respect to the quark fields, one obtains:

$$
G\left(x_{1}, x_{2} ; x_{1}^{\prime}, x_{2}^{\prime}\right)=-\left\langle\operatorname{tr}_{c} U\left(x_{2}, x_{1}\right) S_{1}\left(A ; x_{1}, x_{1}^{\prime}\right) U\left(x_{1}^{\prime}, x_{2}^{\prime}\right) S_{2}\left(A ; x_{2}^{\prime}, x_{2}\right)\right\rangle_{A},
$$


where $S_{1}(A)$ and $S_{2}(A)$ are the quark and antiquark propagators in the presence of the external gluon field and $\operatorname{tr}_{c}$ designates the trace with respect to the color group. The quark propagator $S(A)$ satisfies the equation

$$
\left(i \gamma \cdot \partial_{x}-m-g \gamma \cdot A(x)\right) S\left(A ; x, x^{\prime}\right)=i \delta^{4}\left(x-x^{\prime}\right)
$$

At this stage, the Green function $G$ can schematically be represented as in Fig. 11.

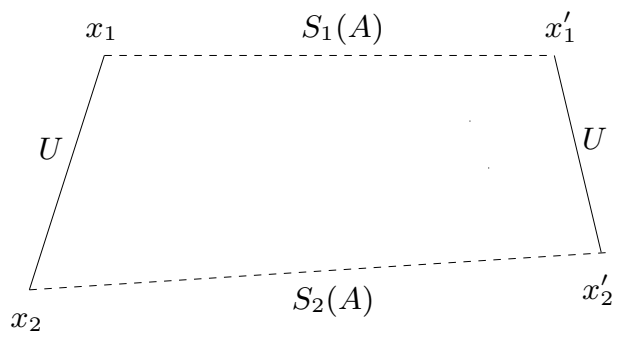

Figure 1: Schematic representation of the two-particle Green function.

In order to make the Wilson loop structure of $G$ apparent, we adopt for the quark propagator in the external gluon field a representation based on an explicit use of the phase factor along straight lines [7]. Introducing the gauge covariant composite object $\widetilde{S}\left(A ; x, x^{\prime}\right)$, made of a free fermion propagator $S_{0}\left(x-x^{\prime}\right)$ (without color group content) multiplied by the path-ordered phase factor $U\left(x, x^{\prime}\right)$ [Eq. (5)] taken along the straight segment $x^{\prime} x$,

$$
\widetilde{S}\left(A ; x, x^{\prime}\right) \equiv S_{0}\left(x-x^{\prime}\right) U\left(x, x^{\prime}\right)
$$

one shows that the quark propagator $S\left(A ; x, x^{\prime}\right)$ in the external gluon field satisfies the following functional integral equation in terms of $\widetilde{S}$ :

$$
S\left(A ; x, x^{\prime}\right)=\widetilde{S}\left(A ; x, x^{\prime}\right)-\int d^{4} x^{\prime \prime} S\left(A ; x, x^{\prime \prime}\right) \gamma^{\alpha} \int_{0}^{1} d \lambda(1-\lambda) \frac{\delta}{\delta x^{\alpha}(\lambda)} \widetilde{S}\left(A ; x^{\prime \prime}, x^{\prime}\right),
$$

where the segment $x^{\prime \prime} x^{\prime}$ has been parametrized with the parameter $\lambda$ as $x(\lambda)=(1-\lambda) x^{\prime \prime}+$ $\lambda x^{\prime}$ and where the operator $\delta / \delta x^{\alpha}(\lambda)$ acts on the factor $U$ of $\widetilde{S}$, along the internal part of the segment $x^{\prime \prime} x^{\prime}$, with $x^{\prime}$ held fixed. That equation is diagrammatically represented in Fig. 2]

A similar equation in which the roles of $x$ and $x^{\prime}$ are interchanged also holds. Those equations lead to iteration series for $S$ in which the gauge covariance property is maintained at each order of the iteration.

Use of the above representations for the quark propagators in Eq. (6) leads for the two-particle Green function to a series expansion where each term contains a Wilson loop 


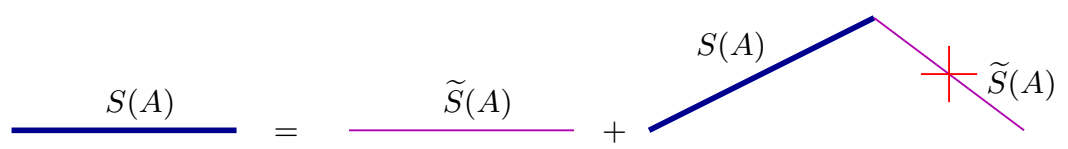

Figure 2: Diagrammatic representation of the integral equation satisfied by the quark propagator in the external gluon field. The cross represents the action of the functional derivative $\delta / \delta x(\lambda)$.

along a skew-polygon:

$$
G=\sum_{i, j=1}^{\infty} G_{i, j},
$$

where $G_{i, j}$ represents the contribution of the term of the series having $(i-1)$ points of integration between $x_{1}$ and $x_{1}^{\prime}\left(i\right.$ segments) and $(j-1)$ points of integration between $x_{2}$ and $x_{2}^{\prime}$ ( $j$ segments). We designate by $C_{i, j}$ the contour associated with the term $G_{i, j}$. A typical configuration for the contour of $G_{4,3}$ is represented in Fig. 3 .

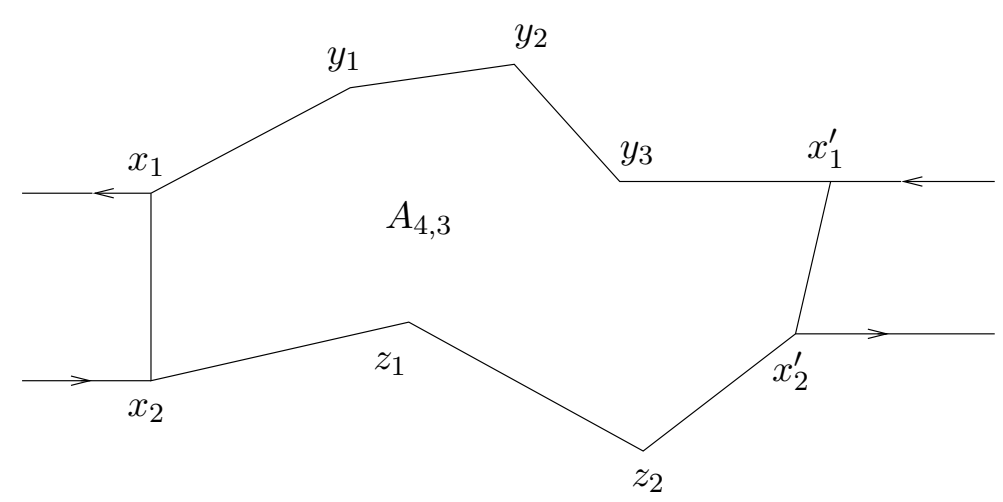

Figure 3: Contour $C_{4.3}$ associated with the term $G_{4,3} . A_{4,3}$ is the minimal surface with contour $C_{4,3}$.

Each segment of the quark lines supports a free quark propagator and except for the first segments (or the last ones, depending on the representation that is used) the Wilson loop is submitted to one functional derivative on each such segment. One then uses for the averages of the Wilson loops appearing in the above series the representation with minimal surfaces [Eq. (3)] .

The Green function $G$ satisfies the following equation with respect to the Dirac operator of particle 1 acting on $x_{1}$ :

$$
\left(i \gamma . \partial_{\left(x_{1}\right)}-m_{1}\right) G\left(x_{1}, x_{2} ; x_{1}^{\prime}, x_{2}^{\prime}\right)=-i\left\langle\operatorname{tr}_{c} U\left(x_{2}, x_{1}\right) \delta^{4}\left(x_{1}-x_{1}^{\prime}\right) U\left(x_{1}^{\prime}, x_{2}^{\prime}\right) S_{2}\left(x_{2}^{\prime}, x_{2}\right)\right\rangle_{A}
$$




$$
-i \gamma^{\alpha}\left\langle\operatorname{tr}_{c} \int_{0}^{1} d \sigma(1-\sigma) \frac{\delta U\left(x_{2}, x_{1}\right)}{\delta x^{\alpha}(\sigma)} S_{1}\left(x_{1}, x_{1}^{\prime}\right) U\left(x_{1}^{\prime}, x_{2}^{\prime}\right) S_{2}\left(x_{2}^{\prime}, x_{2}\right)\right\rangle_{A},
$$

where the segment $x_{1} x_{2}$ has been parametrized with the parameter $\sigma$ as $x(\sigma)=(1-$ $\sigma) x_{1}+\sigma x_{2}$; furthermore, the operator $\delta / \delta x^{\alpha}$ does not act on the explicit boundary point $x_{1}$ of the segment, this contribution having been cancelled by the contribution of the gluon field $A$ coming from the quark propagator $S_{1}$. A similar equation also holds with the Dirac operator of particle 2 acting on $x_{2}$. Representation (9) for the quark propagator can then be used in the above equation and its partner satisfied by the two-particle Green function $G$. One obtains two compatible equations for $G$ where the right-hand sides involve the series of the terms $G_{i, j}$ of Eq. (10) and their functional derivative along the segment $x_{1} x_{2}$. In order to obtain bound state equations, it is necessary to reconstruct in the right-hand sides the bound state poles contained in $G$ [9]. In $x$-space, bound states are reached by taking the large separation time limit between the pair of points $\left(x_{1}, x_{2}\right)$ and $\left(x_{1}^{\prime}, x_{2}^{\prime}\right)$ [10. To produce a bound state pole, it is necessary that there be a coherent sum of contributions coming from each $G_{i, j}$, since the latter, taken individually, do not have poles. Each $G_{i, j}$ involves a corresponding minimal surface $A_{i, j}$ on the contour of which act various functional derivatives. Those can be classified according to their possible irreducibility properties. Reducible contributions are those which are parts of the definition of the series of $G$. It does not seem possible to sum all these terms to reproduce exactly $G$ with some kernel acting on it in the right-hand sides. However, for large separation time limits one can isolate terms that contribute to the pole terms. One notices that the derivative along the segment $x_{1} x_{2}$ acts on areas $A_{i, j}$ with contour $C_{i, j}$ which are different from one term of the series to the other (the number of segments being different). To have a coherent sum of those contributions it is necessary to expand each such derivative term around the derivative of the lowest-order contour $C_{1,1}$, represented in Fig. 4.

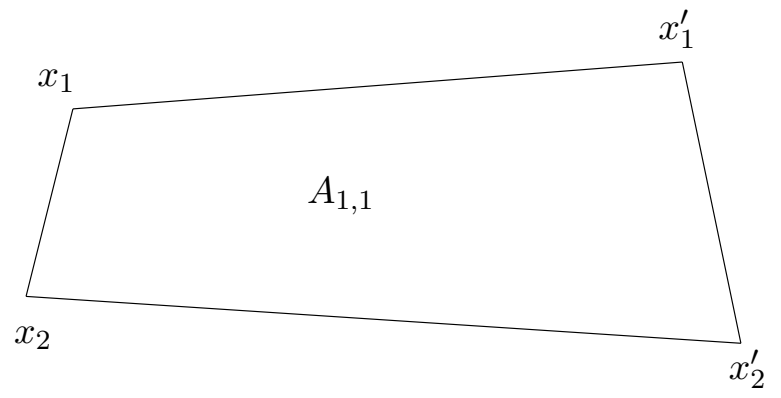

Figure 4: The lowest-order contour $C_{1,1}$ and its minimal surface $A_{1,1}$.

It is that term that can be factorized and can lead through the summation of the 
factored series to the reappearance of the Green function $G$ and to its poles. The remaining terms do not lead to pole terms. Similarly, two derivative contributions should be expanded around the lowest-order contribution coming from the contours $C_{2,1}$ or $C_{1,2}$, and so forth.

In general, the derivative of the areas along $x_{1} x_{2}$ depends among others on the slope of the areas in the orthogonal direction to $x_{1} x_{2}$. One then associates that slope with the quark momenta. Taking then the large separation time limit and equal times in the center-of-mass frame, one ends up with a covariant three-dimensional equation, having the structure of a Breit-Salpeter type equation [11, 12] and where the interaction kernels or potentials are given by various functional derivatives involving at least one derivative along the segment $x_{1} x_{2}$. Keeping for the potentials the terms containing one functional derivative of the area $A_{1,1}$ [Fig. 1], the equation takes the form [7]

$$
\left[P_{0}-\left(h_{10}+h_{20}\right)-\gamma_{10} \gamma_{1}^{\mu} A_{1 \mu}-\gamma_{20} \gamma_{2}^{\mu} A_{2 \mu}\right] \psi(\mathbf{x})=0
$$

where $\psi$ is a $4 \times 4$ matrix wave function of the relative coordinate $x=x_{2}-x_{1}$ considered at equal times, $P_{0}$ the center-of-mass total energy and $h_{10}$ and $h_{20}$ the quark and antiquark Dirac hamiltonians; the Dirac matrices of the quark (with index 1) act on $\psi$ from the left, while the Dirac matrices of the antiquark (with index 2) act on $\psi$ from the right. The potentials $A_{1}$ and $A_{2}$ are defined through the equations

$$
A_{1 \mu}=\sigma \int_{0}^{1} d \sigma^{\prime}\left(1-\sigma^{\prime}\right) \frac{\delta A_{1,1}}{\delta x^{\mu}\left(\sigma^{\prime}\right)}, \quad A_{2 \mu}=\sigma \int_{0}^{1} d \sigma^{\prime} \sigma^{\prime} \frac{\delta A_{1,1}}{\delta x^{\mu}\left(\sigma^{\prime}\right)}
$$

$x\left(\sigma^{\prime}\right)$ belonging to the segment $x_{1} x_{2}$.

The time components of $A_{1}$ and $A_{2}$ add up in the wave equation. For their sum, one has the expression (in the c.m. frame)

$$
\begin{aligned}
& A_{10}+A_{20}=\sigma r \frac{E_{1} E_{2}}{E_{1}+E_{2}}\left\{\left(\frac{E_{1}}{E_{1}+E_{2}} \epsilon\left(p_{10}\right)+\frac{E_{2}}{E_{1}+E_{2}} \epsilon\left(p_{20}\right)\right)\right. \\
& \times \sqrt{\frac{r^{2}}{\mathbf{L}^{2}}}\left(\arcsin \left(\frac{1}{E_{2}} \sqrt{\frac{\mathbf{L}^{2}}{r^{2}}}\right)+\arcsin \left(\frac{1}{E_{1}} \sqrt{\frac{\mathbf{L}^{2}}{r^{2}}}\right)\right) \\
& \left.+\left(\epsilon\left(p_{10}\right)-\epsilon\left(p_{20}\right)\right)\left(\frac{E_{1} E_{2}}{E_{1}+E_{2}}\right)\left(\frac{r^{2}}{\mathbf{L}^{2}}\right)\left(\sqrt{1-\frac{\mathbf{L}^{2}}{r^{2} E_{2}^{2}}}-\sqrt{1-\frac{\mathbf{L}^{2}}{r^{2} E_{1}^{2}}}\right)\right\} .
\end{aligned}
$$

Here, $r=\sqrt{\mathbf{x}^{2}}, E_{a}=\sqrt{m_{a}^{2}+\mathbf{p}^{2}}, a=1,2$, with $m_{a}$ the quark masses, $\mathbf{p}$ the c.m. momentum, $\mathbf{p}=\left(\mathbf{p}_{2}-\mathbf{p}_{1}\right) / 2$, $\mathbf{L}$ the c.m. orbital angular momentum, and $\epsilon\left(p_{10}\right)$ and $\epsilon\left(p_{20}\right)$ the energy sign operators of the free quark and the antiquark, respectively:

$$
\epsilon\left(p_{a 0}\right)=\frac{h_{a 0}}{E_{a}}, \quad a=1,2 .
$$


The space components of $A_{1}$ and $A_{2}$ are orthogonal to $\mathbf{x}$. The expression of $\mathbf{A}_{1}$ is (in the c.m. frame):

$$
\begin{aligned}
\mathbf{A}_{1}=- & \sigma r \frac{E_{1} E_{2}}{E_{1}+E_{2}}\left\{\frac{r^{2}}{2 \mathbf{L}^{2}} \frac{E_{1} E_{2}}{E_{1}+E_{2}} \mathbf{p}^{t}\right. \\
& \times \sqrt{\frac{r^{2}}{\mathbf{L}^{2}}}\left(\arcsin \left(\frac{1}{E_{2}} \sqrt{\frac{\mathbf{L}^{2}}{r^{2}}}\right)+\arcsin \left(\frac{1}{E_{1}} \sqrt{\frac{\mathbf{L}^{2}}{r^{2}}}\right)\right) \\
+ & \frac{1}{E_{2}} \mathbf{p}^{t}\left(\frac{E_{1} E_{2}}{E_{1}+E_{2}}\right)\left(\frac{r^{2}}{\mathbf{L}^{2}}\right)\left(\sqrt{1-\frac{\mathbf{L}^{2}}{r^{2} E_{2}^{2}}}-\sqrt{1-\frac{\mathbf{L}^{2}}{r^{2} E_{1}^{2}}}\right) \\
& \left.-\frac{1}{2} \mathbf{p}^{t}\left(\frac{r^{2}}{\mathbf{L}^{2}}\right)\left(\frac{E_{1}}{E_{1}+E_{2}} \sqrt{1-\frac{\mathbf{L}^{2}}{r^{2} E_{2}^{2}}}+\frac{E_{2}}{E_{1}+E_{2}} \sqrt{1-\frac{\mathbf{L}^{2}}{r^{2} E_{1}^{2}}}\right)\right\} .
\end{aligned}
$$

Here, $\mathbf{p}^{t}$ is the transverse part of $\mathbf{p}$ with respect to $\mathbf{x}$ :

$$
\mathbf{p}^{t}=\mathbf{p}-\mathrm{x} \frac{1}{\mathrm{x}^{2}} \mathrm{x} \cdot \mathrm{p}
$$

The expression of $\mathbf{A}_{2}$ is obtained from that of $\mathbf{A}_{1}$ by an interchange in the latter of the indices 1 and 2 and a change of sign of $\mathbf{p}^{t}$.

For sectors of quantum numbers where $\mathbf{L}^{2}=0$, the expressions of the potentials become:

$$
\begin{aligned}
& A_{10}+A_{20}=\frac{1}{2}\left(\epsilon\left(p_{10}\right)+\epsilon\left(p_{20}\right)\right) \sigma r \\
& \mathbf{A}_{1}=-\frac{1}{E_{1} E_{2}}\left(\frac{1}{3}\left(E_{1}+E_{2}\right)-\frac{1}{2} E_{1}\right) \mathbf{p}^{t} \sigma r \\
& \mathbf{A}_{2}=+\frac{1}{E_{1} E_{2}}\left(\frac{1}{3}\left(E_{1}+E_{2}\right)-\frac{1}{2} E_{2}\right) \mathbf{p}^{t} \sigma r
\end{aligned}
$$

The potentials are generally momentum dependent operators and necessitate an appropriate ordering of terms.

From the structure of the wave equation (12) and the expressions of the potentials, one deduces that the interaction is confining and of the vector type. However, compared to the conventional timelike vector potential, it has additional pieces of terms contributing to the orbital angular momentum dependent parts. A closer analysis of those terms shows that they can be interpreted as being originated from the moments of inertia of the segment $x_{1} x_{2}$ carrying a constant linear energy density equal to the string tension. The interaction potentials are therefore provided by the energy-momentum vector of the segment joining the quark to the antiquark, in similarity with the color flux tube picture of confinement. An analogous equation had also been proposed by Olsson et al. on the basis of a model where the quarks are attached at the ends of a straight string or a color flux tube [13, 14]. A similar conclusion had also been reached by Brambilla, Prosperi et al. on the basis of 
the analysis of the relativistic corrections to the nonrelativistic limit of the Wilson loop [15. 16, 17.

For heavy quarks, one can expand equation (12) around the nonrelativistic limit and obtain the hamiltonian to order $1 / c^{2}$ [7].

The relativistic corrections to the interquark potential arising from the Wilson loop

were analyzed and evaluated in the literature by Eichten and Feinberg [18, Gromes [19, Brambilla, Prosperi et al. [15, 16, 17, Brambilla, Pineda, Soto and Vairo [20].

The Wilson loop approach was also used for the study of quarkonium systems by Dosch, Simonov et al. with the use of the stochastic vacuum model [21].

In conclusion, the saturation of the Wilson loop averages in the large- $N_{c}$ limit by minimal surfaces provides a systematic tool for investigating the large-distance dynamics of quark-antiquark bound state systems.

Acknowledgements: This work was supported in part for H.S. by the EU RTN network EURIDICE under contract No. CT2002-0311.

\section{References}

[1] K. G. Wilson, Phys. Rev. D 10 (1974) 2445.

[2] A. M. Polyakov, Nucl. Phys. B164 (1979) 171.

[3] Yu. M. Makeenko and A. A. Migdal, Phys. Lett. 88B (1979) 135; 97B (1980) 253; Nucl. Phys. B188 (1981) 269.

[4] A. A. Migdal, Phys. Rep. 102 (1983) 199.

[5] Yu. Makeenko, Large N gauge theories, hep-th/0001047.

[6] G. 't Hooft, Nucl. Phys. B72 (1974) 461.

[7] F. Jugeau and H. Sazdjian, Nucl. Phys. B 670 (2003) 221.

[8] V. A. Kazakov and I. K. Kostov, Nucl. Phys. B176 (1980) 199; V. A. Kazakov, Nucl. Phys. B179 (1981) 283.

[9] E. E. Salpeter and H. A. Bethe, Phys. Rev. 84 (1951) 1232.

[10] M. Gell-Mann and F. Low, Phys. Rev. 84 (1951) 350.

[11] G. Breit, Phys. Rev. 34 (1929) 553; 36 (1930) 383; 39 (1932) 616.

[12] E. E. Salpeter, Phys. Rev. 87 (1952) 328.

[13] M. G. Olsson and K. Williams, Phys. Rev. D 48 (1993) 417.

[14] D. LaCourse and M. G. Olsson, Phys. Rev. D 39 (1989) 2751; C. Olson, M. G. Olsson and K. Williams, Phys. Rev. D 45 (1992) 4307; C. Olson and M. G. Olsson, Phys. Rev. D 49 (1994) 4675. 
[15] A. Barchielli, E. Montaldi and G. M. Prosperi, Nucl. Phys. B296 (1988) 625; B303 (1988) 752 (E); A. Barchielli, N. Brambilla and G. M. Prosperi, Nuovo Cimento 103 A (1990) 59.

[16] N. Brambilla, P. Consoli and G. M. Prosperi, Phys. Rev. D 50 (1994) 5878; N. Brambilla, E. Montaldi and G. M. Prosperi, Phys. Rev. D 54 (1996) 3506.

[17] M. Baldicchi and G. M. Prosperi, Phys. Lett. B 436 (1998) 145; Phys. Rev. D 62 (2000) 114024; 66 (2002) 074008.

[18] E. Eichten and F. Feinberg, Phys. Rev. D 23 (1981) 2724.

[19] D. Gromes, Z. Phys. C 22 (1984) 265; 26 (1984) 401.

[20] A. Pineda and A. Vairo, Phys. Rev. D 63 (2001) 054007; 64 (2001) 039902 (E); N. Brambilla, A. Pineda, J. Soto and A. Vairo, Phys. Rev. D 63 (2001) 014023.

[21] H. G. Dosch, Phys. Lett. B 190 (1987) 177; Yu. A Simonov, Nucl. Phys. B307 (1988) 512; H. G. Dosch and Yu. A. Simonov, Phys. Lett. B 205 (1988) 339; A. Di Giacomo, H. G. Dosch, V. I. Shevchenko and Yu. A. Simonov, Phys. Rep. 372 (2002) 319. 